\title{
On the Flexible 5G Dense Deployment Air Interface for Mobile Broadband
}

\author{
Eeva Lähetkangas, Kari Pajukoski, Jaakko Vihriälä, Esa Tiirola \\ Nokia Networks, Oulu, Finland
}

\begin{abstract}
In this paper we contemplate 5G frame structure for dense deployment. We discuss harmonized OFDM concept, in which the cyclic prefix length and subcarrier spacing are functions of carrier frequency while FFT size and base clock remain constant. The subframe structure consists of time separated control and data, enabling low latency, flexible UL/DL ratio switching and high spectral efficiency with low cost transceiver. Analysis shows that the overheads are relatively small. The subframe structure also allows extended sleep periods, enabling considerably reduced power consumption.
\end{abstract}

Index Terms-3GPP, 5G, energy consumption, frame structure, harmonized OFDM, latency, local area, LTE, RAT, subcarrier spacing, TDD, TTI.

\section{INTRODUCTION}

5G Radio Access, targeted to be available beyond 2020, is expected to handle a very wide range of requirements and use cases, including among others mobile broadband (MBB) and machine type communications (MTC). For MBB, 5G radio access is expected to fulfill the demand of exponentially increasing data traffic and to allow people and machines to enjoy gigabit data rates with virtually zero latency. Also, $5 \mathrm{G}$ is expected to reduce energy consumption and costs compared to the existing $4 \mathrm{G}$ technologies. To realize this vision of ubiquitous mobile broadband, dense deployments with access to very large bandwidths are required. This leads us to consider a wide range of frequency bands, varying from below $6 \mathrm{GHz}$ area up to the millimeter wave $(\mathrm{mmW})$ frequencies. In order to reduce implementation and hardware cost and to provide inbuilt support e.g. for relaying, the same $5 \mathrm{G}$ air interface should be suited not only for traditional access links but also for new connection types, such as self-backhauling and possibly also e.g. device-to-device links [1]. The bursty and variant traffic of the connection types leads to the need of efficient spectrum usage and resource management. Thus, since spectrum flexibility can be considered to be more important than coverage, time division duplexing (TDD) is seen as a more attractive duplexing method over frequency division duplexing (FDD) in 5G dense deployment environment. The rest of the paper focuses on TDD mode.

The large increase of $5 \mathrm{G}$ data throughput leads to the need of transmitting and processing of larger amounts of data, consequently imposing demands on baseband processing. A baseband system can cope with the increased throughput demand by decreasing the latency. Thus, besides the achievement of high data rates, also latency reductions at the air interface level become vital in order to enable e.g. energy savings and long battery life time. Latency is also especially important in a scheduled TDD system where several TDD cycles are required for delivering one round trip transmission related to control signaling or data transmission. On air interface level, these requirements essentially lead to the demand of fast link direction switching and to short transmission time interval (TTI) length, further leading to the transmission of shorter blocks of data in time and wider blocks in frequency.

The selection of the radio waveform is an important discussion topic in the design of $5 \mathrm{G}$ radio access, due to its impact on transceiver design, complexity and the radio numerology. So far, Orthogonal Frequency Division Multiplexing (OFDM) and DFT-spread (precoded) OFDM, as well as Filter Bank Multicarrier (FBMC), have been most widely considered. The waveforms to be used in $5 \mathrm{G}$ radio access should cope with a set of $5 \mathrm{G}$ requirements, such as high spectral efficiency at least for sub-millimeter-wave frequencies, low latency and limited cost and complexity.

Good time localization is required in order to enable low latency. Especially in adaptive TDD systems with the need of fast and flexible link direction switching, the waveform needs to enable short switching guard times between TDD link directions. Further, in order to enable usage of low cost hardware chips, the computational complexity related to the waveform needs to be limited. In general it can be stated that FBMC overcomes OFDM in terms of reduced overhead and spectral containment, but with the cost of higher complexity and poor time localization. The OFDM outband emission problem becomes negligible in dense deployment environment due to the usage of low power levels with very wide channel bandwidths. Co-channel interference can be seen as the major limiting factor instead of interference due to adjacent channels. Also, the good time-localization properties of OFDM waveform make it optimal from latency perspective. Very large MIMO and high gain beamforming provide energy efficient and cost efficient MBB solution, especially in higher frequency bands. Though recent research on FBMC has shown promising improvements, the feasibility of FBMC with practical implementations remains for further research, including the effect of nonlinear amplification leading to the reduction of FBMC spectral properties. For these reasons, we consider OFDM based waveforms to be most suited for $5 \mathrm{G}$ MBB technology over FBMC and focus to OFDM based system in the rest of the paper.

The air interface flexibility and latency of TDD Long Term Evolution Advanced (LTE-A) [2][3] are limited by its physical frame structure. The hard limit for the air interface latency in LTE-A is set by the design with a restriction of including at maximum two uplink (UL) / downlink (DL) 
switching points inside one $10 \mathrm{~ms}$ LTE-A radio frame. Evolutions of LTE-A will also not be able to support full flexibility nor major latency reductions due to the restrictions of incremental evolution and backwards compatibility reasons.

In this paper, we present the main concept of $5 \mathrm{G}$ air interface optimized for $5 \mathrm{G}$ small cell environment and feasible for a wide range of frequencies. First, we introduce and discuss a concept of harmonized OFDM, in which OFDM numerology is flexibly scaled according to the carrier frequency. We then present a TDD optimized 5G subframe structure functioning on top of this numerology and discuss the enhanced properties it enables compared to existing technologies. Further, we provide a more detailed analysis on how the properties of the proposed subframe structure can further be utilized to achieve extremely low energy consumption and compare the obtained $5 \mathrm{G}$ battery life time estimations to the corresponding limits of LTE.

The paper is structured as follows. In Section II, we introduce and discuss the concept of harmonized OFDM. In Section III, we describe the proposed 5G TDD subframe structure. Section IV provides an analysis of wake up latency and its relationships to power consumption. Finally, Section V concludes the paper.

\section{HARMONIZED OFDM}

For OFDM, when increasing carrier frequency from $\mathrm{GHz}$ towards $\mathrm{mmW}$ range, it is proposed simultaneously to increase the used bandwidth and subcarrier (SC) spacing, while keeping the FFT size within a small set of quantized values. Smaller cell sizes lead to decreased propagation losses, while delay spread may also be expected to decrease when moving towards higher carrier frequencies with larger bandwidth. Thus, similar scaling can be done in time domain numerology, meaning that e.g. the delay spread and the related numerology, such as cyclic prefix $(\mathrm{CP})$ length, may further be variable according to the carrier frequency. The principle of scalable radio numerology is referred here as harmonized OFDM and is illustrated in Fig. 1 with some preliminary numerology values.

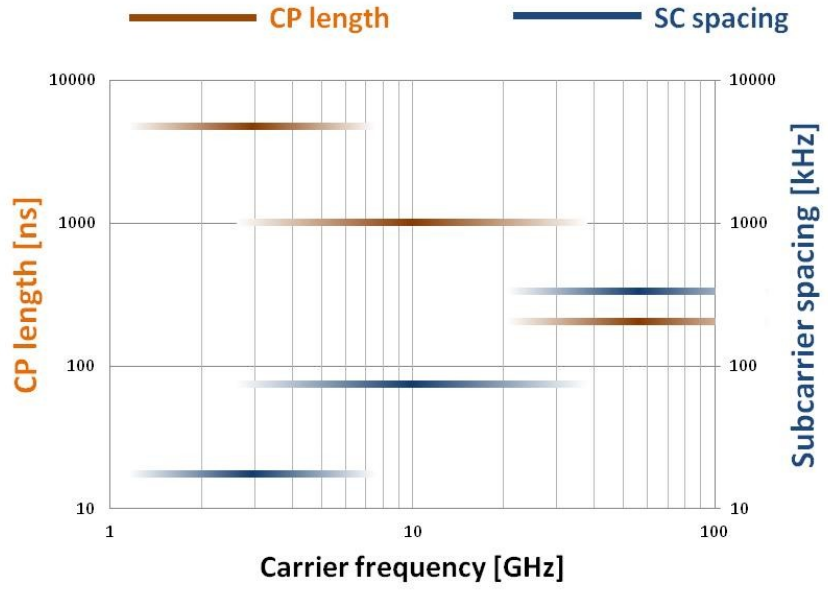

Fig. 1. Harmonized OFDM concept.

For more detailed analysis of the values of different time domain steps in Fig. 1, we first consider the impact of CP. In
[4], it has been estimated that a CP length of about $1 \mu$ s would be feasible for $5 \mathrm{G}$ dense deployments with centimeter wave carrier frequency. In order to analyze this in more detail, the spectral efficiency performances per layer for two channel Power Delay Profiles (PDPs), namely ITU indoor hotspot (InH) NLoS and ITU urban macro (UMa) NLoS, were calculated. Results are shown in Figures 2 and 3 for several SC spacing values. Results for ITU urban micro (UMi) NLoS would lie in between InH and UMa. We use $40 \mathrm{~dB}$ channel SNR (including error vector magnitude (EVM)) and maximum modulation of 256-QAM with rate 0.9 , which gives $S E_{\max }=$ $7.2 \mathrm{bps} / \mathrm{Hz}$. These results are shown to illustrate the maximum achievable spectral efficiency taking into account the effect of SNR degradation due to too short CP (using the method described in [5]), and overhead from the CP [6].

By comparing the difference of the optimal spectral efficiency values to the spectral efficiency achieved with $1 \mu \mathrm{s}$ $\mathrm{CP}$ length in Figures 2 and 3 with $60 \mathrm{kHz} \mathrm{SC}$ spacing, we notice that the degradation in spectral efficiency (SE) performance is at maximum about 5-6\%. Thus, to overcome the channel delay spread, constant $1 \mu \mathrm{s}$ CP length seems to be sufficient for all investigated channel models. Timing alignment (TA) has been used in macro and large cells to compensate the propagation delay. Thus, with TA, we can conclude that $1 \mu \mathrm{s}$ CP length should be feasible for channels varying from indoor hotspot to outdoor micro and macro, causing less than $6 \%$ overhead with $60 \mathrm{kHz}$ SC spacing.

\section{InH NLoS}

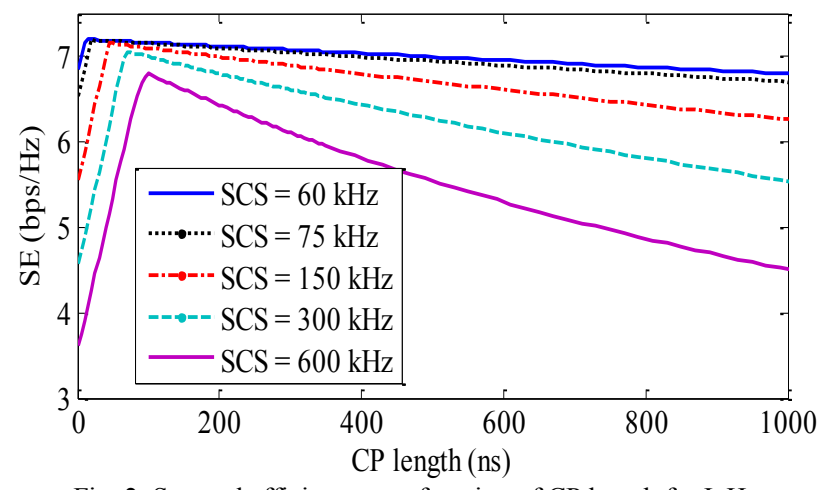

Fig. 2. Spectral efficiency as a function of CP length for InH.

UMa NLoS

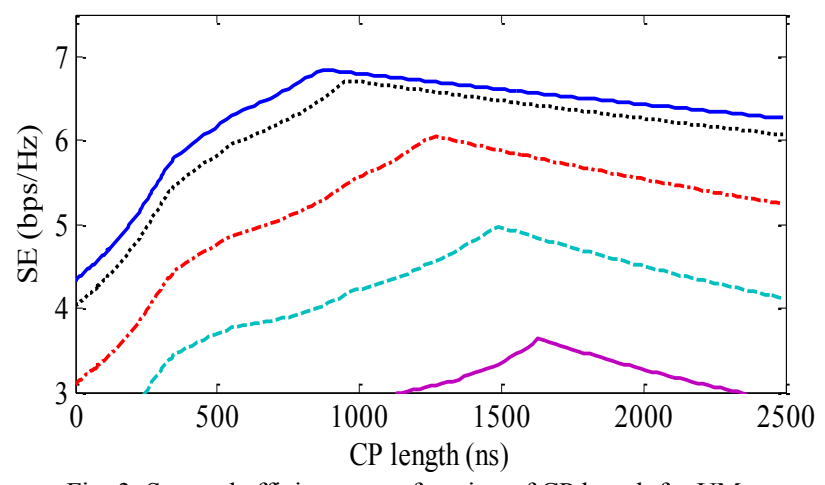

Fig. 3. Spectral efficiency as a function of CP length for UMa. 
Next, we extend the analysis to include also the impact of Doppler effect causing inter-carrier interference (ICI) in OFDM systems together with phase noise $(\mathrm{PN})$, caused by implementation technology and hardware design. In multicarrier systems, the effect of PN depends on the subcarrier spacing [7]. Fig. 4 shows the preliminary analysis of optimal subcarrier spacing and $\mathrm{CP}$ length for two frequencies and with cell radius of $10 \mathrm{~m}: 6 \mathrm{GHz}$ (for which we use InH and UMa channels), and $60 \mathrm{GHz}$ (802.11ad conference room). Jointly optimal values for the CP length and SC spacing (SCS) are found for a SNR of $16 \mathrm{~dB}$, taking into account phase noise, Doppler spread at $50 \mathrm{~km} / \mathrm{h}$ velocity and a nominal EVM of $40 \mathrm{~dB}$ due to other imperfections. The measured PN PSD of a phase-locked loop $90 \mathrm{~nm}$ CMOS oscillator for $\sim 37.6 \mathrm{GHz}$ [8] is used as a basis to roughly estimate the PN effect at $6 \mathrm{GHz}$ and $60 \mathrm{GHz}$, by shifting the PN PSD by $-20 \mathrm{dBc} / \mathrm{Hz}$ per decade of decrease in the carrier frequency.

This scaling of PN PSD gives a very rough estimate, leading a more detailed analysis still for further study. The PN ICI is calculated according to the formulas given in [9] and over bandwidths of $2 \mathrm{GHz}$ and $200 \mathrm{MHz}$ for $60 \mathrm{GHz}$ and 6 $\mathrm{GHz}$ carrier frequencies respectively. Only PN ICI term is analyzed, meaning that the PN common phase error (CPE) term is not taken into consideration, since it can be assumed to be sufficiently corrected with pilots provided that the subcarrier spacing is larger than the significant part of the CPE. Also further ICI compensation algorithms are not considered. The results of this preliminary analysis presented in Fig. 4 indicate that the estimated subcarrier spacing values even for $\mathrm{mmW}$ frequency area seem still to be reasonably low. Slight increasing of the SC spacing and adding some pilots for PN estimation and compensation may still be required in order to take for example CPE and different RF impairments into account, leading to a small additional further increase in the overhead. Still, together with much shorter required CP length the overhead can be estimated to stay still in feasible limits.

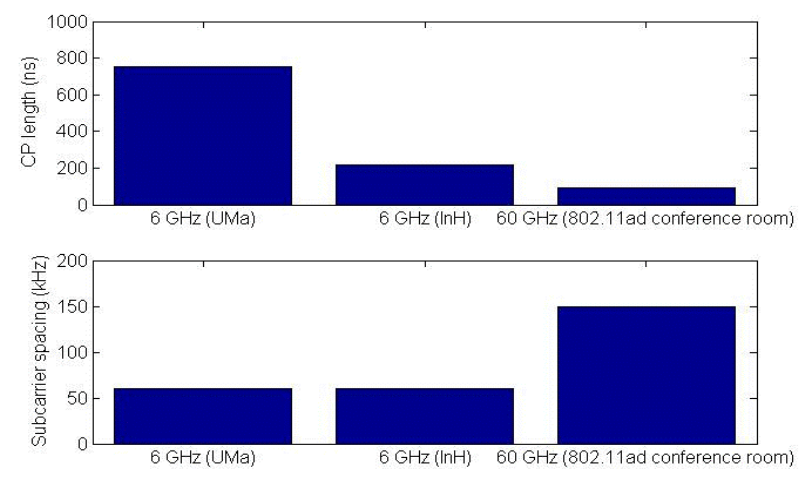

Fig. 4. Optimal CP length and subcarrier spacing.

\section{5G SUBFRAME STRUCTURE}

Deployment densification with smaller cell sizes and utilization of higher carrier frequencies with larger bandwidth provide remarkable enablers for optimization of air interface and leading to reduced air interface latency. The characteristics of local area environment together with certain expected improvements in component technology within the 5G timeframe, such as shorter TDD link direction hardware switching time, will eventually provide possibility for a TDD numerology optimized for dense deployment, e.g. shorter cyclic prefix and guard period (GP) times compared to the existing systems [4]. Further, short OFDM symbols correspond to large subcarrier spacing, which is robust to the increased oscillator phase noise at higher carrier frequencies. The properties listed above can be utilized to design a $5 \mathrm{G}$ physical frame structure for dense deployments, for which the time and frequency numerology is scalable according to harmonized OFDM principle presented in Section II. In [4], we proposed a TDD optimized subframe structure, for which the principle is illustrated in Fig. 5. Only one TDD type is presented here, in the other TDD mode the transmission (TX) and receiving $(\mathrm{RX})$ phases are in the opposite order.

In order to enable robust and fast control plane, control signaling is embedded to each subframe and time separated from the data plane. Control symbols are also located before data symbols in order to allow fast and cost efficient pipeline processing at the receiver. Control part of the subframe provides possibility to include both RX and TX control resources, allowing the devices in the network to both receive and send control signals, such as scheduling requests and grants, in every subframe. In addition to the scheduling related control information, control plane may also contain reference signals (RS), used for cell detection and selection, scheduling in frequency domain, precoder selection and channel estimation. Rest of the subframe after the control part is dedicated for data plane. In order to achieve low complexity, data part in one subframe contains only transmitting or receiving. In [4] and [6], it was analyzed that very short subframe lengths, such as $0.25 \mathrm{~ms}$, are feasible with the proposed subframe structure from the guard and control overhead point of view, using relatively low $60 \mathrm{kHz}$ subcarrier spacing. Short subframe length can further be utilized to enable short physical layer round trip time with fast control signaling and fast TDD data switching periodicity.

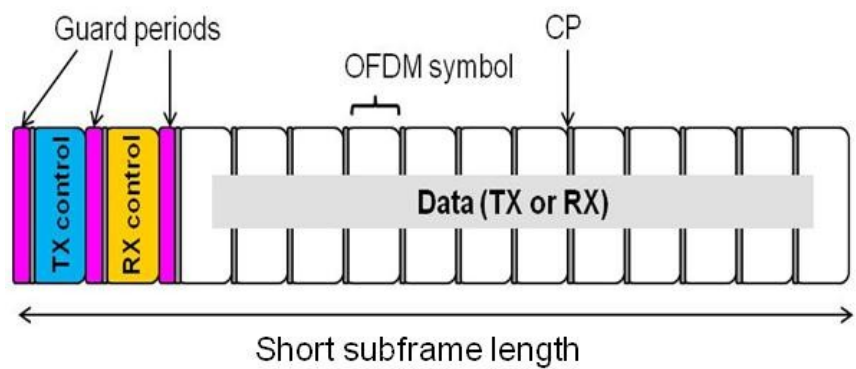

Fig. 5. 5G physical TDD subframe structure for dense deployments.

The 5G physical TDD subframe structure with short subframe length enables multiple properties providing gain with respect to the existing technologies. These properties and the provided flexibility fit well not only for access links but also for other communication types in small cell environment, 
such as backhaul, device-to-device and machine type links. Possibility to allocate data part of the subframe either for TX or RX enables fully flexible UL/DL ratio switching for data transmission. The decreased guard overheads, such as GP and $\mathrm{CP}$, together with flexibility in UL/DL ratio improve the maximum achievable link spectral efficiency (LSE) per link direction compared to TDD LTE-A. Since it is possible to embed a synchronization signal (e.g. by eNB or AP) to the control part of each subframe, it is possible to enable very fast network synchronization by the network devices. The proposed subframe structure also allows fast and robust control signalling and handshakes, such as related to UE initiated data transmission [4.] These properties can be utilized to achieve decreased energy consumption, like explained in more detail in Section IV. Further, the subframe structure allows (UL/DL) symmetric demodulation reference signal (DMRS) design. DMRS can be embedded e.g. to the first symbols in data part and since the DMRS symbols between link directions are fully aligned, it is possible to design crosslink orthogonal reference signals. Thus, the proposed subframe structure contains inbuilt support for cross-link interference mitigation, further enabling usage of advanced receivers.

In addition, the simplified control plane and its presence in each subframe enables a clean TDD hybrid automatic repeat request (HARQ) scheme with HARQ timing not dependent on the UL/DL ratio. HARQ timing can be fixed and counted in subframes, which remarkably reduces TDD HARQ complexity and decreases the related HARQ latency with respect to TDD LTE-A. It can be shown that the total HARQ round trip time (RTT) enabled by the proposed subframe structure is $\leq \sim 1 \mathrm{~ms}$, which is a clear improvement compared e.g. to corresponding value of LTE-A [4]. A reduced HARQ latency further enables usage of fewer HARQ processes, reducing also memory consumption and device cost since less receiver HARQ buffers are required. Since every subframe has control in both directions, control signalling of HARQ can be made independent of the direction of the data part. The enabled HARQ arrangement is therefore also well-suited for multi-hop scenarios.

\section{REDUCED ENERGY CONSUMPTION}

Reduced physical layer latency and properties of physical subframe structure presented in Section III can be utilized to reduce energy consumption of the network devices. Measurements on LTE smartphones [10] have shown that the power consumption ratio between the UE being $\mathrm{ON}$ and sleeping is at least 1:35. Maximizing the UE's sleep time is therefore essential to improve the battery life. The power consumption perspective of the procedure where UE wakes up from the low-power sleep mode to receive or transmit data is illustrated in Fig. 6. This procedure can be divided into five different states: sleeping, waking up (wup), synchronizing, transferring data, and powering down (pd). When the power up/down states when changing from sleep to active mode and vice versa are device dependent, the time duration of the synchronization and transfer states are directly related to the latency and frame length of the wireless standard.

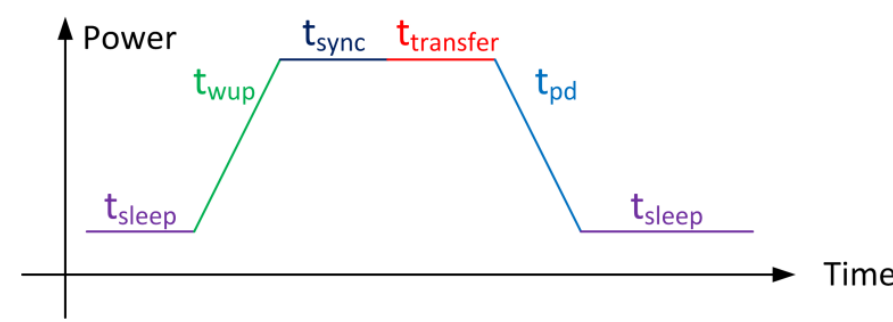

Fig. 6. UE power states.

Due to the shorter subframes and the possibility to allocate synchronization signals in every subframe, the $5 \mathrm{G}$ UE will be able to synchronize much faster compared to e.g. LTE UE with significantly longer synchronization time. The time to transfer data in 5G will also be shorter compared to LTE due to the increased data rates reducing the actual transfer time. Furthermore, the shorter 5G subframe length enables reduction of the total time required for the transmission of scheduling request (SR) and scheduling grant (SG). In the $5 \mathrm{G}$ concept, due to the bidirectional control plane embedded to each subframe, the UE initiated data transmission/reception [6] can be achieved within 5 subframes +1 symbol as illustrated in Fig. 7. The total time required for this procedure is estimated to be $\sim 1.27 \mathrm{~ms}$ (with $0.25 \mathrm{~ms}$ subframe length) or even shorter with shorter subframe length.

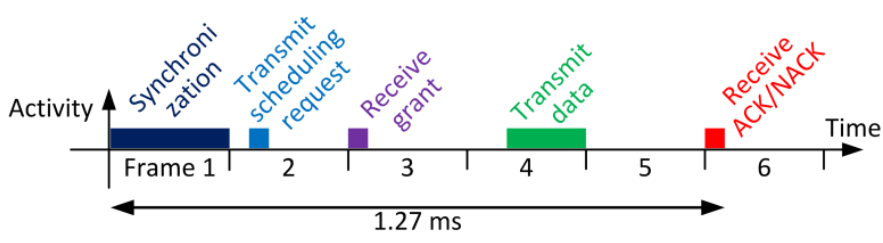

Fig. 7. Procedure for UE initiated transmission in $5 \mathrm{G}$.

The short physical layer latency enabling fast transitions between sleep and active modes can be utilized to bring significant gains in terms of power consumption. Fig. 8 shows results of theoretical numerical calculations of the battery life for $5 \mathrm{G}$ system as a function of reception opportunity times and the number of transmissions per second [6]. Corresponding results for machine-to-machine (M2M)-optimized TDD LTE [11] are also presented for comparison. The results have been generated assuming same power consumption values [11] and utilization of $32400 \mathrm{Ws}$ low cost battery for both technologies. In the transmission phase, the $5 \mathrm{G} \mathrm{UE}$ is assumed to follow the UE initiated transmission procedure according to Fig. 7. In the reception phase, the UE is assumed to do only synchronization and thereafter to receive a short paging message with the length of one OFDM symbol. The analysis results in Fig. 8 show that compared to M2M optimized TDD LTE design, approximately 7-40 times lower energy consumption can be achieved with the proposed $5 \mathrm{G}$ concept. 


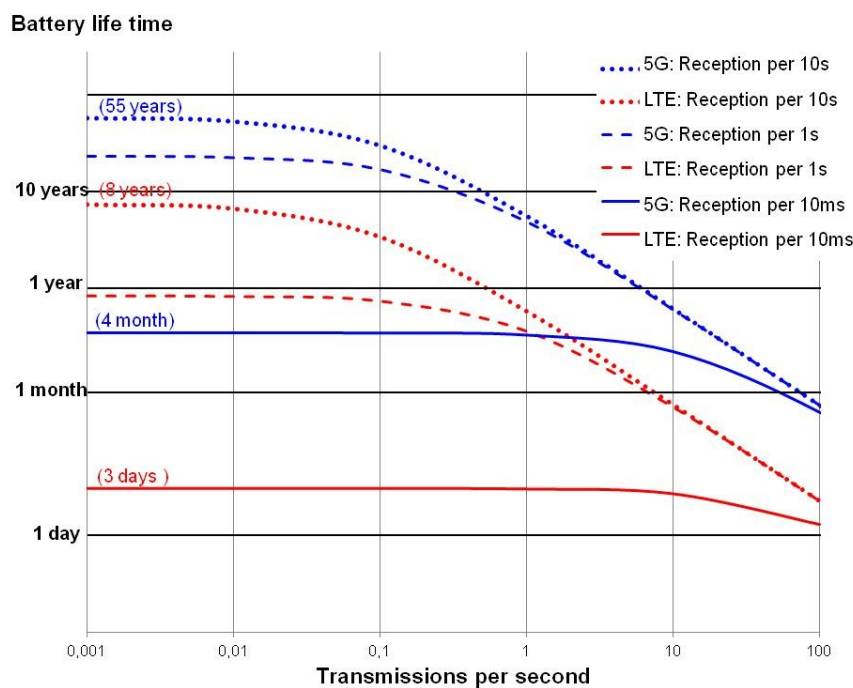

Fig. 8. Battery life time comparison for M2M optimized LTE and 5G.

\section{CONCLUSIONS}

In this paper, we discussed the concept of dense deployment optimized $5 \mathrm{G}$ air interface for mobile broadband. We presented the idea of harmonized OFDM numerology, enabling the usage of same baseband design for wide area of frequencies by flexibly scaling the OFDM numerology according to used carrier frequency.

TDD optimized 5G subframe structure for small cell environment designed on top of this numerology was also presented together with discussion of various enabled benefits provided by it over the existing technologies, such as LTE. The properties enabled by the proposed subframe structure include among others full flexibility in terms of UL/DL ratio switching, fast network synchronization, short physical layer latency, enabled usage of advanced receivers and support for low-complex HARQ schemes.

Special emphasis was put to latency and energy consumption aspects. The proposed $5 \mathrm{G}$ subframe structure with very short subframe length enables really short UE initiated data transmission/reception times, enabling network devices to remain in energy-efficient sleep mode for majority of time and consequently leading to very long battery life. A numerical analysis shows that with the same power and battery assumptions the proposed $5 \mathrm{G}$ concept has approximately 7-40 times lower energy consumption compared to M2M optimized TDD LTE.

\section{ACKNOWLEDGEMENTS}

Part of this work has been performed in the framework of the FP7 project ICT-317669 METIS, which is partly funded by the European Union. The authors would like to acknowledge the contributions of their colleagues in METIS, although the views expressed are those of the authors and do not necessarily represent the project.

\section{REFERENCES}

[1] P. Mogensen, K. Pajukoski, B. Raaf, E. Tiirola, E. Lähetkangas, I.Z Kovács, G. Berardinelli, L.G.U. Garcia, L. Hu, A.F. Cattoni, "B4G local area: high level requirements and system design", IEEE Globecom, 2012.

[2] 3GPP TS 36.211, Evolved Universal Terrestrial Radio Access (EUTRA); Physical channels and modulation, Available: www.3gpp.org.

[3] 3GPP TS 36.213 v.10.8.0, Physical layer procedures for Evolved Universal Terrestrial Radio Access (E-UTRA). Available: www.3gpp.org.

[4] E. Lähetkangas, K. Pajukoski, E. Tiirola, G. Berardinelli, I. Harjula, J. Vihriälä, "On the TDD Subframe Structure for Beyond 4G Radio Access Network", Future Network \& Mobile Summit, July 2013.

[5] M. Batariere, K. Baum, and T. Krauss, "Cyclic Prefix Length Analysis for 4G OFDM Systems," Proc. of IEEE Vehicular Technology Conference (VTCFall), CA, USA, pp. 543-547, 2004.

[6] E. Lähetkangas, K. Pajukoski, J. Vihriälä et al., "Achieving low latency and energy consumption by 5G TDD mode optimization", IEEE ICC2014, June 10-14, Sydney, Australia, 2014.

[7] T. Pollet, M. Van Bladel, and M. Moeneclaey, "BER sensitivity of OFDM systems to carrier frequency offset and Wiener phase noise," IEEE Trans. Commun., Volume: 43, Issue: 2/3/4, 1995.

[8] J. Lee, M. Liu, H. Wang: “A 75-GHz phase-locked loop in 90-nm CMOS technology”, IEEE J. Solid-State Circuits, vol. 43, 2008.

[9] IEEE 802.16.3c-01/49, "Performance aspects of OFDM PHY proposal", available in http://wirelessman.org/tg3/contrib/802163c01_49.pdf.

[10] M. Lauridsen, L. Noël, T.B. Sørensen, P. Mogensen, “An Empirical LTE Smartphone Power Model with a View to Energy Efficiency Evolution”, Intel Technology Journal vol. 18 issue 1, March 2014.

[11] T. Tirronen, A. Larmo, J. Sachs, B. Lindoff, N. Wiberg, "Reducing Energy Consumption of LTE Devices for Machine-to-Machine Communication", Globecom Workshops (GC Wkshps), December 2012. 